\title{
Actively tunable thin films for visible light by thermo-optic modulation of $\mathrm{ZnO}$
}

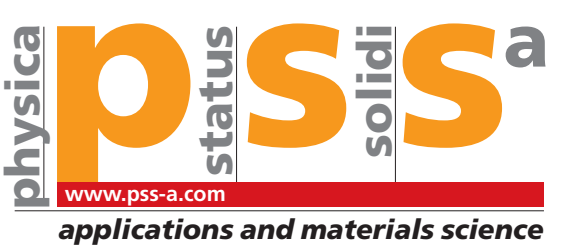

\author{
Enes Battal ${ }^{*, 1}$ and Ali Kemal Okyay ${ }^{*, 1,2,3}$ \\ ${ }^{1}$ Department of Electrical and Electronics Engineering, Bilkent University, Ankara 06800, Turkey \\ ${ }^{2}$ UNAM - National Nanotechnology Research Center, Bilkent University, Ankara 06800, Turkey \\ ${ }^{3}$ Institute of Materials Science and Nanotechnology, Bilkent University, Ankara 06800, Turkey
}

Received 4 June 2015, revised 29 November 2015, accepted 22 December 2015

Published online 23 January 2016

Keywords antireflection coatings, ellipsometry, Fabry-Perot resonance, modulators, thermo-optical materials, zinc oxide

* Corresponding author: e-mail enesbattal@gmail.com, Phone: +90 3122903525, Fax: +90 3122901219

** e-mail aokyay@ee.bilkent.edu.tr, Phone: +90 3122901557, Fax: +90 3122901219

Applications of active control of light matter interactions within integrated photonics, hyper-spectral imaging, reconfigurable lasers, and selective bio-surfaces have enormously increased the demand for realization of optical modulation covering the spectrum from ultraviolet (UV) up to infrared (IR) wavelength range. In this study, we demonstrate $\mathrm{ZnO}$-based actively tunable perfect absorber operating within UV and visible spectrum with more than $5 \mathrm{~nm}$ shift in the resonant absorption wavelength. Using spectroscopic ellipsometry technique, we extract temperature-dependent optical constants of atomic layer-deposited $\mathrm{ZnO}$ within $0.3-1.6$ and 4-40 $\mu \mathrm{m}$ spectra. We also observe bandgap narrowing of $\mathrm{ZnO}$ at elevated temperatures due to lattice relaxation verified by the red-shift of phonon-modes. At around its bandgap, refractive index variations up to 0.2 is obtained and $\mathrm{ZnO}$ is shown to exhibit thermo-optic coefficient as high as $9.17 \times 10^{-4} \mathrm{~K}^{-1}$ around the bandgap which is the largest among well-known large bandgap materials.
1 Introduction Reconfigurable optical structures are desired to increase the control over the light matter interaction especially due to expansion of optoelectronic applications within optical computation and communication, display, lighting, imaging, holographic technologies. Conventionally, free-carrier dispersion, electro-absorption, electro-optic and thermo-optic effects are among the dominantly exploited methods for realization of optical modulation. However, the limitations of these effects in terms of loss, modulation contrast, speed, bandwidth, spectral coverage as well as integration cost drive the efforts toward development of new schemes. Recently, novel active optical tuning schemes have been introduced by coupling novel optical resonators such as metamaterials [1], plasmonic gratings [2], optical cavities [3], and photonic crystals [4] with conventionally used refractive index varying media such as silicon [5], GaAs [6], $\mathrm{LiNbO}_{3}$ [7], liquid crystals [8]. Recent advancements in wide bandgap materials-based optoelectronics, especially aimed for visible and ultraviolet spectrum, have put more emphasis on realization of integrated actively reconfigurable devices. Among large bandgap materials, $\mathrm{ZnO}$ which has a large band gap of $3.37 \mathrm{eV}$ [9] and exciton binding energy of $60 \mathrm{meV}$ [9], has attracted significant attention due to its potential in ultraviolet laser [10,11], photodetection [12], and also infrared imaging [13] applications.

In this study, we introduce a $\mathrm{ZnO}$-based actively reconfigurable thin film utilizing thermo-optic effect. By forming a Fabry-Perot cavity structure, we obtain perfect absorption at resonant wavelengths and actively modulate the resonant wavelength by varying the temperature between 23 and $200{ }^{\circ} \mathrm{C}$. We observe more than $5 \mathrm{~nm}$ shift within ultraviolet and visible spectra. We investigate thermal dependence of refractive index values of $\mathrm{ZnO}$ in 300 $1600 \mathrm{~nm}$ spectrum using spectroscopic ellipsometry method. Due to thermal relaxation under thermal stress, we observe red-shift in the optical bandgap of the $\mathrm{ZnO}$ which provides higher modulation in the refractive index around the bandedge. In addition, we also investigate the optical constants of $\mathrm{ZnO}$ within IR spectrum, 4-40 $\mu \mathrm{m}$ and reflection spectra of the structure; and no significant modulation in the reflection is observed. We also verified the thermal relaxation within $\mathrm{ZnO}$ thin film through observation of red-shift in the optical phonon modes of $\mathrm{ZnO}$. 
2 Ellipsometric characterization We start with the characterization of temperature-dependent optical properties of $\mathrm{ZnO}$ film in the UV-VIS-NIR and Mid-IR region through spectroscopic ellipsometry method. This method utilizes the change in the polarization of the light upon reflection characterized by complex reflectance ratio $\rho=r_{\mathrm{p}} / r_{\mathrm{s}}=\tan (\Psi) \mathrm{e}^{j \Delta}$, where $\Psi$ and $\Delta$ are the measured amplitude ratio and phase difference between $\mathrm{p}$ - and s-polarized light. Measurements are carried out on a FabryPerot resonant cavity structure formed by coating a 240-nmthick $\mathrm{ZnO}$ layer on top of a p-type silicon wafer having resistivity in the range of $0.1-0.9 \Omega \mathrm{cm}$. Standard RCA cleaning procedure is carried out before film deposition. Using atomic layer deposition technique, $\mathrm{ZnO}$ is coated through 1700 deposition cycles within Cambridge Savannah 100 chamber at $250^{\circ} \mathrm{C}$ using diethylzinc (DEZ) and milli-Q water $\left(\mathrm{H}_{2} \mathrm{O}\right)$ as reaction precursors.

We performed the measurements using commercial ellipsometers V-Vase and IR-Vase by J. A. Woollam Co., for UV-VIS-NIR and Mid-IR wavelengths, respectively. Before the measurements, the samples are annealed in atmoshperic conditions at $250{ }^{\circ} \mathrm{C}$ for $90 \mathrm{~min}$. Temperature-dependent measurements are carried out by cycling the sample temperature between 22 and $200^{\circ} \mathrm{C}$ while illuminating at an angle of incidence of $55^{\circ}$ and $57^{\circ}$ at UV-VIS-NIR and Mid-IR wavelengths, respectively. A nonlinear least square error fitting algorithm has been used to fit oscilator parameters to measured $\Psi / \Delta$ values and the mean square error of the data fit remained below 3 for all temperatures. In order to eliminate the effects of thermo-optic modulation of optical constants of $\mathrm{Si}$, we extracted optical constants of bare substrate at the temperatures of interest through employing point by point fit and we used these values as reference while fitting the optical constants for $\mathrm{ZnO}$.

We used a Cody-Lorentz oscillator coupled with two Gaussian oscillators and an undamped Lorentz oscillator (a pole) to model the dielectric permittivity of $\mathrm{ZnO}$. CodyLorentz oscillator model is widely used for modeling wide bandgap semiconductors and includes the contributions from defect states as well as intraband absorptions which would result in nonzero absorption below the bandgap in the form of an exponentially decaying function named as Urbach's Tail [14]. The resulting equations for the dielectric function are given as follows:

$$
\begin{aligned}
& \epsilon^{\prime \prime}{ }_{\text {Gauss }}(E)=A \mathrm{e}^{-\left(\frac{\ln \left(E-E_{0}\right)}{\Gamma}\right)^{2}}-A \mathrm{e}^{-\left(\frac{\ln 4\left(E+E_{0}\right)}{\Gamma}\right)^{2}}, \\
& \epsilon^{\prime \prime}{ }_{\text {Cody-Lorentz }}(E)=\left\{\begin{array}{c}
\frac{E_{1}}{E} \mathrm{e}^{\frac{\left(E-E_{\mathrm{H}}\right)}{E_{\mathrm{u}}}}, E<\left(E_{\mathrm{g}}-E_{\mathrm{t}}\right) \\
\frac{\left(E-E_{\mathrm{g}}\right)^{2}}{\left(E-E_{\mathrm{g}}\right)^{2}+E^{2}} \frac{A E_{\mathrm{o}} \Gamma E}{\left(E^{2}-E^{2}\right)^{2}+\Gamma^{2} E^{2}}, E>\left(E_{\mathrm{g}}-E_{\mathrm{t}}\right),
\end{array}\right.
\end{aligned}
$$

$$
\epsilon^{\prime \prime}(E)=\epsilon^{\prime \prime}{ }_{\text {Cody-Lorentz }}(E)+\epsilon^{\prime \prime}{ }_{\text {Gauss-1 }}(E)+\epsilon^{\prime \prime}{ }_{\text {Gauss }-2}(E),
$$

$$
\begin{aligned}
& \epsilon_{\text {Pole }}^{\prime}(E)=\frac{A}{E_{\mathrm{o}}^{2}-E^{2}}, \\
& \epsilon^{\prime}(E)=\epsilon_{\infty}+\epsilon_{\text {Pole }}^{\prime}(E)+\frac{2}{\pi} P \int_{-\infty}^{\infty} \frac{\xi \epsilon^{\prime \prime}(\xi)}{\xi^{2}-E^{2}} \mathrm{~d} \xi \\
& \epsilon(E)=\epsilon^{\prime}(E)+j \epsilon^{\prime \prime}(E) .
\end{aligned}
$$

By summing two Gaussian oscillators described in Eq. (1) with a Cody-Lorentz oscillator described in Eq. (2), the imaginary part of the dielectric function of $\mathrm{ZnO}$ is formed in Eq. (3). Adding the undamped Lorentz oscillator in Eq. (4), the static dielectric constant $\left(\varepsilon_{\infty}\right)$, and KramersKronig Transform of the imaginary part of the dielectric function of $\mathrm{ZnO}$ results in the real part of the dielectric function of $\mathrm{ZnO}$ in Eq. (5). The complex representation of the dielectric function of $\mathrm{ZnO}$ is written in Eq. (6). In these equations, $E$ stands for photon energy, $E_{\mathrm{o}}$ stands for the center resonance frequency of the oscillator, $\Gamma$ is the broadening parameter, $A$ is the oscillator amplitude, $E_{\mathrm{g}}$ is the optical bandgap, $E_{\mathrm{p}}$ is the transition energy separating absorption onset from Lorentzian behavior, $E_{\mathrm{t}}$ is the transition energy between Urbach tail and band-to-band transitions, $E_{\mathrm{u}}$ is the Urbach Energy defining the spectral rate of decay of the absorption, and $E_{1}$ is a fit parameter providing continuity at $E=E_{\mathrm{t}}$. The extracted parameters are given in Table 1 as a function of temperature. Optical bandgap of the coated $\mathrm{ZnO}$ film is extracted to be about $3.23 \mathrm{eV}$ at room-temperature and increase in the temperature causes lowering of the bandgap from 3.23 to $3.10 \mathrm{eV}$ for temperature modulation from 22 to $200^{\circ} \mathrm{C}$. The lowering of the bandgap of a semiconductor is predicted by Varshni's formulation [15] based on increase in the inter-atomic distance due to thermal expansion.

The corresponding temperature-dependent refractive index of $\mathrm{ZnO}$ calculated from $n=\operatorname{Re}\{\sqrt{\epsilon}\}$ in $300-1600 \mathrm{~nm}$ range are shown in Fig. 1a. Throughout the spectrum, modulation of refractive index is present in both real and imaginary parts. Such large spectral coverage of thermo-optic effect in the $\mathrm{ZnO}$ thin film is similar to that of well-known large bandgap materials, $\mathrm{AlN}, \mathrm{GaN}, \mathrm{SiC}$ [11]. The modulation is repeatable and volatile such that the refractive index values of $\mathrm{ZnO}$ returns to its initial values after the thermal stress is removed. Maximum modulation is observed at around the bandgap of $\mathrm{ZnO}$ as shown in Fig. 1b. Variation of refractive index up to 0.2 is observed which results in a thermo-optic coefficient of $9.17 \times 10^{-4} \mathrm{~K}^{-1}$.

This value is more than four times higher compared to the thermo-optic coefficients of well-known wide bandgap materials such as $\mathrm{AlN}, \mathrm{GaN}, \mathrm{SiC}[16,17]$ at around their bandgap. In addition, the sharp peak observed at the bandedge of the extinction spectra of $\mathrm{ZnO}$ in Fig. 1c can be attributed to the involvement of light-exciton interactions in the dielectric function as exciton binding energy of $\mathrm{ZnO}$, $60 \mathrm{meV}$, is large enough to make photogenerated bound excitons to be distinguishable near the bandedge. The 
Table 1 Oscillator parameters for the temperature-dependent optical properties of $\mathrm{ZnO}$ in $300-1600 \mathrm{~nm}$ spectrum.

\begin{tabular}{|c|c|c|c|c|c|c|c|c|c|c|c|c|c|}
\hline \multirow[b]{2}{*}{$T\left({ }^{\circ} \mathrm{C}\right)$} & \multirow[b]{2}{*}{$\epsilon_{\infty}$} & \multicolumn{2}{|c|}{$\epsilon_{\text {Pole }}^{\prime}$} & \multicolumn{7}{|c|}{$\epsilon^{\prime \prime}$ Cody-Lorentz } & \multicolumn{3}{|c|}{$\epsilon^{\prime \prime}$ Gauss-1/2 } \\
\hline & & $A$ & $\begin{array}{l}E_{\mathrm{o}} \\
(\mathrm{eV})\end{array}$ & $A$ & $\begin{array}{l}E_{\mathrm{o}} \\
(\mathrm{eV})\end{array}$ & $\begin{array}{l}\Gamma \\
(\mathrm{eV})\end{array}$ & $\begin{array}{l}E_{\mathrm{g}} \\
(\mathrm{eV})\end{array}$ & $\begin{array}{l}E_{\mathrm{p}} \\
(\mathrm{eV})\end{array}$ & $\begin{array}{l}E_{\mathrm{t}} \\
(\mathrm{eV})\end{array}$ & $\begin{array}{l}E_{\mathrm{u}} \\
(\mathrm{eV})\end{array}$ & $A$ & $\begin{array}{l}E_{\mathrm{o}} \\
(\mathrm{eV})\end{array}$ & $\begin{array}{l}\Gamma \\
(\mathrm{eV})\end{array}$ \\
\hline 23 & 2.24 & 18.3 & 5.35 & 140 & 3.09 & 0.105 & 3.26 & 0.992 & 0.0102 & 1.204 & $0.352 / 1.21$ & $3.443 / 3.38$ & $0.405 / 0.140$ \\
\hline 100 & 2.46 & 14.8 & 5.30 & 196 & 2.68 & 0.147 & 3.16 & 1.003 & 0.0181 & 1.178 & $0.777 / 0.944$ & $3.442 / 3.36$ & $0.358 / 0.121$ \\
\hline 200 & 2.39 & 17.8 & 5.40 & 212 & 2.50 & 0.156 & 3.10 & 1.004 & 0.0191 & 0.883 & $0.738 / 0.714$ & $3.424 / 3.32$ & $0.397 / 0.134$ \\
\hline
\end{tabular}

excitonic peak red-shifts along with the bandgap and also decays significantly when the temperature increases to $200^{\circ} \mathrm{C}$ as expected since the available kinetic energy gets closer to the binding energy of the excitons which weakens the strength observed excitonic resonances. Below the
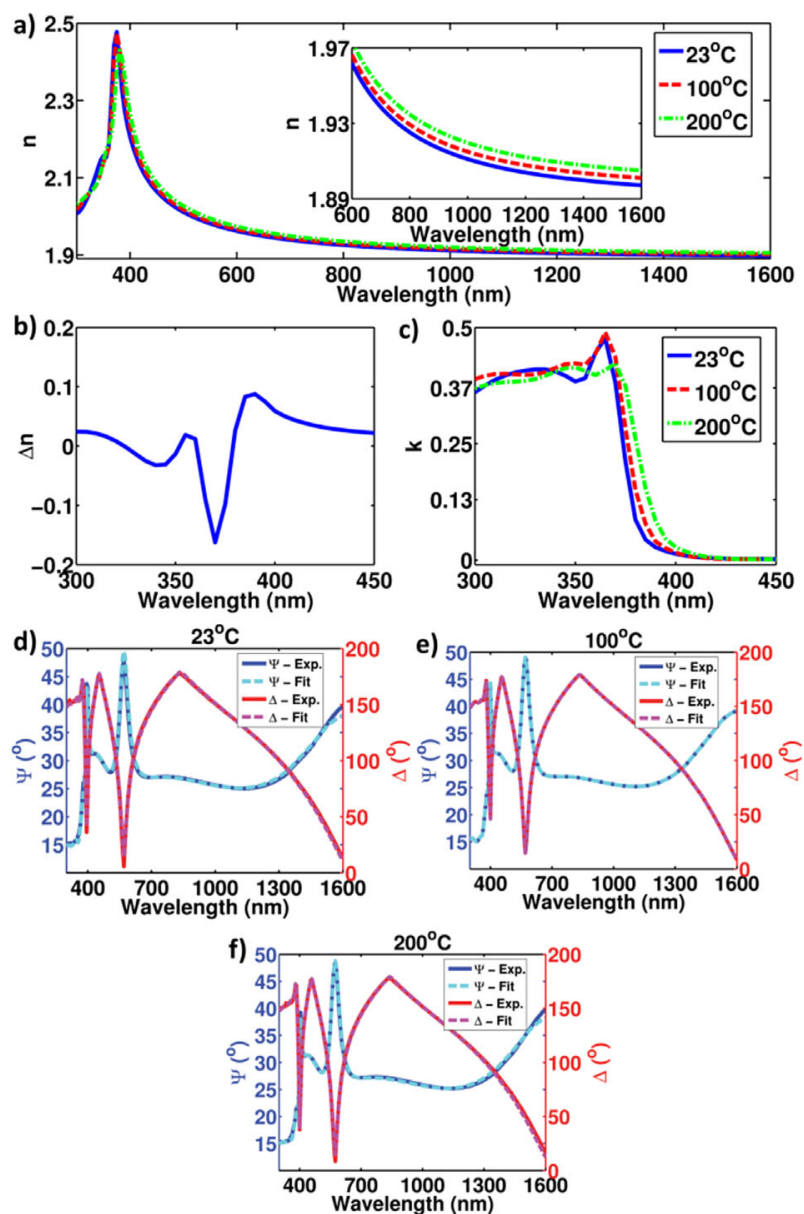

Figure 1 (a) Real part of the refractive index of $\mathrm{ZnO}$ as a function of temperature exhibiting thermo-optic modulation in the entire $300-1600 \mathrm{~nm}$ spectra. The inset zooms into the refractive index in sub-bandgap region. (b) Refractive index difference near the bandgap for increasing temperature from 23 to $200{ }^{\circ} \mathrm{C}$. (c) Imaginary part of the refractive index of $\mathrm{ZnO}$ as a function of temperature shows the red-shift in the bandedge by increased temperature. For temperatures of (d) $23{ }^{\circ} \mathrm{C}$, (e) $100^{\circ} \mathrm{C}$, and (f) $200^{\circ} \mathrm{C}$, raw ellipsometric data and fitting results obtained using Cody-Lorentz, undamped Lorentz, and Gaussian oscillators agree very well. bandgap energy, the modulation of refractive index remains at about 0.01 for temperature difference of $177^{\circ} \mathrm{C}$ as shown in the inset of Fig. 1a. This is an order of magnitude lower compared to the modulation around the bandgap energy.

In addition, decrease in $E_{\mathrm{u}}$ by the elevation of temperature gives an increase in the extinction coefficient of $\mathrm{ZnO}$ by 0.002 as a result of increase in the density of the occupied trap-states. Above the bandgap energy, there is still a refractive index modulation of about 0.02 for the same thermal difference which is attributed to the active modification of density of states due to thermal relaxation. Figure 1d-f shows very good agreement between the raw ellipsometric data and the fitting results for all the temperatures of interest in the UV-VIS-NIR sepectrum.

We also investigated the temperature dependence of optical constants of $\mathrm{ZnO}$ at Mid-IR wavelengths covering 4-40 $\mu \mathrm{m}$ spectrum. Within this spectrum, optical phonon resonances of $\mathrm{ZnO}$ is known to contribute significantly to the dielectric function [18] as well as the unintentional n-type doping of $\mathrm{ZnO}$ which can reach concentrations well above $10^{16} \mathrm{~cm}^{-3}$ [19]. Although the sensitivity of optical constants of $\mathrm{ZnO}$ films to the crystal orientation is minimal in the UV-VIS-NIR region [9], optical phonon modes present in $\mathrm{ZnO}$ films are highly sensitive to crystal orientation $[18,20]$. Hence optical anisotropy is taken into account in the modeling of IR optical constants of $\mathrm{ZnO}$ films. In order to model the dielectric permittivity at this range, we have used a TO-LO oscillators coupled with Drude oscillator to account for the effects of both the phonon modes and free carriers of the grown $\mathrm{ZnO}$ film. While modeling the phonon modes, we have considered the inherent uniaxial anisotropy in $\mathrm{ZnO}$ associated with the polycrystalline nature of the ALD grown $\mathrm{ZnO}$ films. The equations describing the dielectric function of $\mathrm{ZnO}$ film at these wavelengths are as follows:

$$
\begin{aligned}
& \epsilon(E)=\epsilon_{\text {Drude }}(E)+\epsilon_{\mathrm{TO}-\mathrm{LO}}(E), \\
& \epsilon_{\text {Drude }}(E)=-A \frac{\Gamma}{E^{2}+j \Gamma E}, \\
& \epsilon_{\mathrm{TO}-\mathrm{LO}}(E)=A \frac{E_{\mathrm{LO}}^{2}-E^{2}+j \Gamma E}{E_{\mathrm{TO}}^{2}-E^{2}+j \Gamma E},
\end{aligned}
$$

where $E_{\mathrm{LO}}$ and $E_{\mathrm{TO}}$ stands for the longitudinal and transverse phonon oscillation energy, for which the resulting fit parameters are given in Table 2 . The optical constants are 
separated into out-of-plane $(\perp)$ and in-plane (II) parts due to uniaxial anisotropy. Literature values are used for $E_{\mathrm{TO} \perp}$ and $E_{\mathrm{LO}}$ as IR ellipsometry technique becomes insensitive to these parameters for film thicknesses much less than a micrometer. The carrier concentration of the films are extracted to be $1.07 \times 10^{19} \mathrm{~cm}^{-3}$ from Drude relation. This value agrees well with the literature [21]. Resulting temperature-dependent infrared dielectric permittivities are depicted in Fig. 2a-d. Very good fitting to the raw mid-IR ellipsometric data is achieved in the mid-IR spectrum for both 23 and $200{ }^{\circ} \mathrm{C}$ as depicted in Fig. 2e and f, respectively. The optical constants in midIR regime do not exhibit significant modulation with temperature except where the phonon modes of $\mathrm{ZnO}$ are dominant $(\sim 27 \mu \mathrm{m})$. In-plane phonon modes observed at these wavelengths red-shift by $100 \mathrm{~nm}$ when the temperature is increased from room temperature to $200{ }^{\circ} \mathrm{C}$ as a result of increased atomic separation due to thermal expansion. Anisotropy in the optical constants arise due to the presence of phonon modes since the difference between in plane and out of plane values diminish as the wavelengths get closer to the near IR regime.

3 Actively tunable thin film Fabry-Perot (FP) resonance is achieved through forming a constructive interference within an optical cavity. In our case, thin layer of $\mathrm{ZnO}$ on top of $\mathrm{Si}$ acts as an optical cavity such that the reflected wave from $\mathrm{ZnO} / \mathrm{Si}$ interface constructively interferes with both incident wave and the reflected wave from air/ZnO interface, which results in a standing wave within the film as depicted in Fig. 3a. At the resonance wavelengths, the films do not reflect any portion of the incident light, therefore, all the incident light gets absorbed within both $\mathrm{ZnO}$ and $\mathrm{Si}$ layers. The resonance wavelength is defined by the following formula:

$$
R=\left|r_{1}+\frac{t_{1} t_{2} r_{2} \mathrm{e}^{\mathrm{j} 2 n_{\mathrm{ZnO} k d}}}{1-r_{2} r_{3} \mathrm{e}^{-j 2 n_{\mathrm{Zno}} k d}}\right|^{2}
$$

where $r$ and $t$, respectively, corresponds to Fresnel reflection and transmission coefficients at the corresponding boundaries depicted in Fig. 3a. At room temperature $\left(23^{\circ} \mathrm{C}\right)$, the reflection spectrum in UV-VIS-NIR range for $20^{\circ}$ angle of incidence and p-polarization is shown in Fig. $3 \mathrm{~b}$ along with the theoretical calculation using Eq. (10) and the extracted optical constants in Fig. 1a. Experimental reflection is
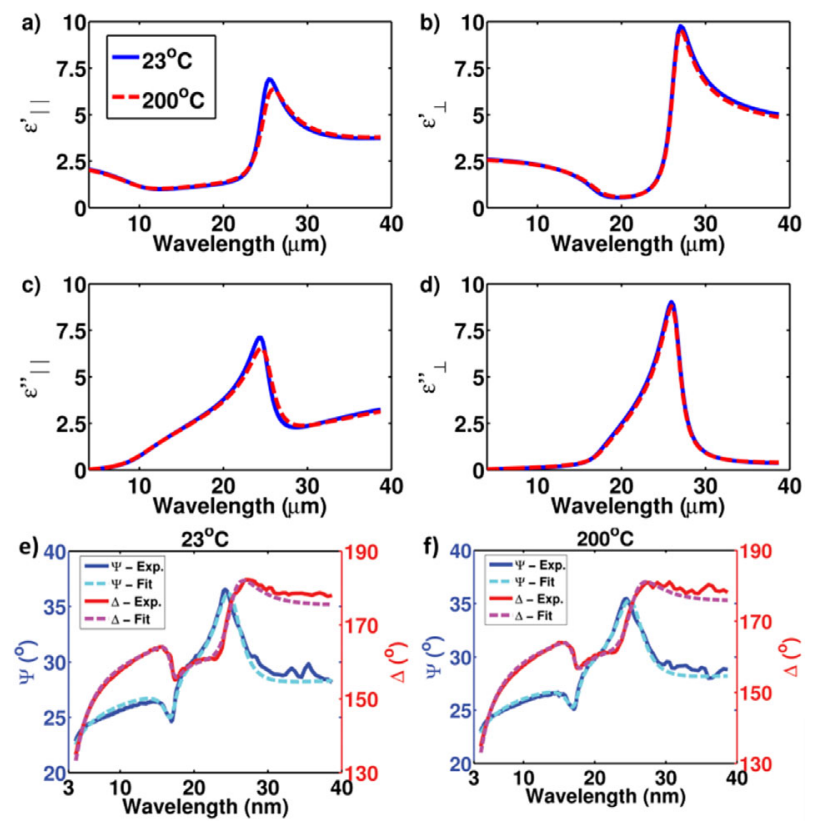

Figure 2 (a), (b) Real and (c), (d) imaginary parts of the midinfrared anisotropic dielectric constants of $\mathrm{ZnO}$ for in-plane and out-of-plane directions, respectively. Thermal modulation causes red-shift for the phonon modes in this spectrum. The mid-IR dielectric function model constructed using TO-LO and Drude oscillators results in very good fitting of the raw ellipsometric data for (e) $23^{\circ} \mathrm{C}$ and (f) $200{ }^{\circ} \mathrm{C}$.

calculated by dividing the reflected light intensity from the sample at an angle of incidence to the background light intensity. Using the extracted optical constants of $\mathrm{ZnO}$, room temperature FP resonances are predicted to occur at the wavelengths of 407 and $610 \mathrm{~nm}$ which match very well with measurements having no reflection at these wavelengths. These wavelengths, 410 and $610 \mathrm{~nm}$, are, respectively, the second and third order FP resonances, formulated by $d=(2 m+1)\left(\lambda / 4 n_{\mathrm{ZnO}}\right)$ where $m$ is an integer. The resonances occur only within the wavelength regime where $\mathrm{ZnO}$ exhibits transparency since thinner films are required in order to form a cavity resonance within the regions showing strong absorption which is above the bandgap for $\mathrm{ZnO}$.

Active tunability of the $\mathrm{ZnO}$ thin film is investigated through reflection spectrum for the same temperature range of interest in Fig. 3c. We extracted reflection spectrum during the rise and fall times of the temperature modulation

Table 2 Oscillator parameters for the temperature-dependent optical constants of $\mathrm{ZnO}$ in $4-40 \mu \mathrm{m}$ spectrum.

\begin{tabular}{|c|c|c|c|c|c|c|c|}
\hline \multirow[b]{2}{*}{$T\left({ }^{\circ} \mathrm{C}\right)$} & \multirow[b]{2}{*}{ anisotropy axis } & \multicolumn{2}{|l|}{$\epsilon_{\text {Drude }}$} & \multicolumn{4}{|l|}{$\epsilon_{\mathrm{TO}-\mathrm{LO}}$} \\
\hline & & $A$ & $\Gamma(\mathrm{eV})$ & $A$ & $E_{\mathrm{LO}}\left(\mathrm{cm}^{-1}\right)$ & $E_{\mathrm{TO}}\left(\mathrm{cm}^{-1}\right)$ & $\Gamma\left(\mathrm{cm}^{-1}\right)$ \\
\hline \multirow[t]{2}{*}{23} & $\|$ & 0.924 & 0.0664 & 4.993 & 590 & 401.7 & 34.22 \\
\hline & $\perp$ & 0.0669 & 0.917 & 6.997 & 584 & 378 & 27.17 \\
\hline \multirow[t]{2}{*}{200} & $\|$ & 0.875 & 0.0702 & 4.855 & 590 & 397.6 & 41.48 \\
\hline & $\perp$ & 0.0772 & 0.795 & 6.824 & 576.5 & 378 & 26.64 \\
\hline
\end{tabular}



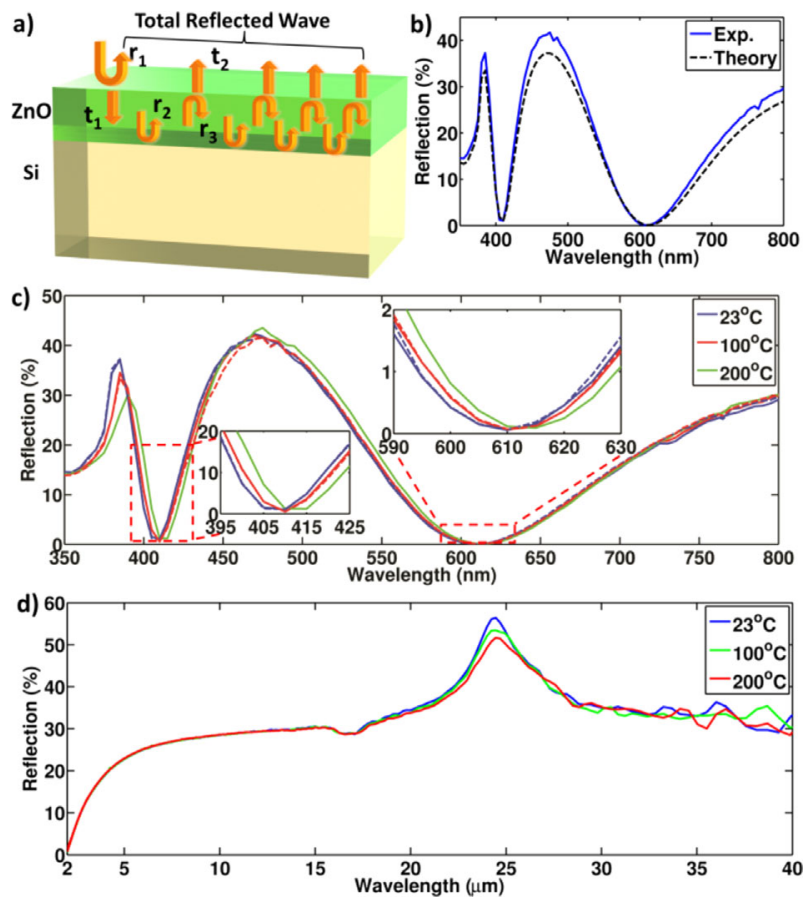

Figure 3 (a) Depiction of Fabry-Perot resonance in the $\mathrm{ZnO} / \mathrm{Si}$ structure showing multiple internal reflectances. (b) The measured reflection spectrum at the angle of incidence of $20^{\circ}$ and ppolarization showing two FP resonances agrees well with the theoretical calculation. (c) FP resonances in reflection spectrum of the $\mathrm{ZnO} / \mathrm{Si}$ structure is modulated by $6 \mathrm{~nm}$ through thermo-optic effect within UV-VIS range. Solid and dashed lines correspond to the reflection spectrum in the rise and fall times, respectively. (d) Reflection spectra in mid-IR wavelengths show no significant modulation except for the red-shift of the phonon modes due to thermal expansion.

which are represented by solid and dashed lines in the corresponding figure. The reflection measurements are performed on the same sample right after the ellipsometric measurements at each temperature step. By increasing the temperature up to $200^{\circ} \mathrm{C}$, the resonance wavelength shifts by $6 \mathrm{~nm}$ for the resonance at UV and visible regime as a result of increase in the refractive index. Although the structure does not result in constructive resonance near the band-edge of the $\mathrm{ZnO}$, the spectral shift of the peak in reflection at these wavelengths is about $10 \mathrm{~nm}$ considerably due to shift in the band gap resulting higher refractive index modulation as mentioned before.

We also performed reflection measurements at $25^{\circ}$ angle of incidence in Mid-IR regime whose results are shown in Fig. 3d. The first order FP-resonance is partially observed near $2 \mu \mathrm{m}$-end of the spectrum with almost zero reflection. FP resonance diminishes for the wavelengths well above $2 \mu \mathrm{m}$ since the structure becomes optically subwavelength and no significant modulation in the reflection spectrum is observed. In this region, only $E_{\mathrm{LO} \perp}$ and $E_{\mathrm{TO}}$ phonon modes extracted to be at about 17.1 and $24.9 \mu \mathrm{m}$ are observable. Increasing the temperature, red-shifts the phonon modes by about $100 \mathrm{~nm}$ consistent with the ellipsometric measurements.

4 Conclusions We demonstrated a ZnO-based actively tunable thin film operating in the UV-VIS wavelengths utilizing thermo-optic effect and FP resonance. Ellipsometric extraction of temperature-dependent optical constants of $\mathrm{ZnO}$ in UV-VIS spectra yielded modulation of refractive index by up to 0.2 for a temperature difference of $177^{\circ} \mathrm{C}$, therefore, a thermo-optic coefficient of $9.17 \times 10^{-4} \mathrm{~K}^{-1}$. This value corresponds to the highest thermo-optic coefficient among the well-known wide bandgap materials [17]. We also extracted temperature dependent optical constants at Mid-IR wavelengths covering $4-40 \mu \mathrm{m}$ range and observed that the phonon modes red-shift at elevated temperature due to thermal expansion. The results of this work pave the way for realization of high performance modulation schemes in applications like integrated modulators, reconfigurable antireflective coatings, color filters, and spatial light modulation.

Acknowledgements This work was supported by the Scientific and Technological Research Council of Turkey (TUBITAK), grant nos. 109E044, 112M004, 112E052, and 113M815. A.K.O. acknowledges support from European Union FP7 Marie Curie International Reintegration Grant (PIOS, grant no. PIRG04-GA-2008-239444). A.K.O. acknowledges support from the Turkish Academy of Sciences Distinguished Young Scientist Award (TUBA GEBIP).

\section{References}

[1] I. M. Pryce, K. Aydin, Y. A. Kelaita, R. M. Briggs, and H. A. Atwater, Nano Lett. 10, 4222-4227 (2010).

[2] Y. J. Liu, Y. B. Zheng, J. Liou, I. K. Chiang, I. C. Khoo, and T. J. Huang, J. Phys. Chem. C 115, 7717-7722 (2011).

[3] Z. Zheng, G. Yang, H. Li, and X. Liu, Opt. Express 19, 2158-2164 (2011).

[4] D. Sridharan, R. Bose, H. Kim, G. S. Solomon, and E. Waks, Opt. Express 19, 5551-5558 (2011).

[5] P. Dong, S. Liao, H. Liang, R. Shafiiha, D. Feng, G. Li, X. Zheng, A. V. Krishnamoorthy, and M. Asghari, Opt. Express 18, 25225-25231 (2010).

[6] J. r. B. Kinzel, D. Rudolph, M. Bichler, G. Abstreiter, J. J. Finley, G. Koblmüller, A. Wixforth, and H. J. Krenner, Nano Lett. 11, 1512-1517 (2011).

[7] R. Takigawa, E. Higurashi, T. Suga, and T. Kawanishi, Opt. Express 19, 15739-15749 (2011).

[8] I. Moreno, J. A. Davis, T. M. Hernandez, D. M. Cottrell, and D. Sand, Opt. Express 20, 364-376 (2012).

[9] Ü. Özgür, Y. I. Alivov, C. Liu, A. Teke, M. A. Reshchikov, S. Doğan, V. Avrutin, S.-J. Cho, and H. Morkoç, J. Appl. Phys. 98, 041301 (2005).

[10] Y. R. Ryu, J. A. Lubguban, T. S. Lee, H. W. White, T. S. Jeong, C. J. Youn, and B. J. Kim, Appl. Phys. Lett. 90, 131115 (2007).

[11] S. Chu, G. Wang, W. Zhou, Y. Lin, L. Chernyak, J. Zhao, J. Kong, L. Li, J. Ren, and J. Liu, Nature Nano 6, 506-510 (2011). 
[12] K. J. Chen, F. Y. Hung, S. J. Chang, and S. J. Young, J. Alloys Compd. 479, 674-677 (2009).

[13] E. Battal, S. Bolat, M. Y. Tanrikulu, A. K. Okyay, and T. Akin, Phys. Status Solidi A 211, 2475-2482 (2014).

[14] M. A. Jacobson, O. V. Konstantinov, D. K. Nelson, S. O. Romanovskii, and Z. Hatzopoulos, J. Cryst. Growth 230, 459-461 (2001).

[15] V. Alex, S. Finkbeiner, and J. Weber, J. Appl. Phys. 79, 6943-6946 (1996).

[16] W. Naoki, K. Tsunenobu, and S. Jun, Jpn. J. Appl. Phys. 51, 112101 (2012).

[17] E. D. Palik (ed.), Thermo-Optic Coefficients, in: Handbook of Optical Constants of Solids (Academic Press, Burlington, 1997), pp. 115-261.
[18] S. Heitsch, C. Bundesmann, G. Wagner, G. Zimmermann, A. Rahm, H. Hochmuth, G. Benndorf, H. Schmidt, M. Schubert, M. Lorenz, and M. Grundmann, Thin Solid Films 496, 234-239 (2006).

[19] C. A. Wolden, T. M. Barnes, J. B. Baxter, and E. S. Aydil, J. Appl. Phys. 97, 043522 (2005).

[20] N. Ashkenov, B. N. Mbenkum, C. Bundesmann, V. Riede, M. Lorenz, D. Spemann, E. M. Kaidashev, A. Kasic, M. Schubert, M. Grundmann, G. Wagner, H. Neumann, V. Darakchieva, H. Arwin, and B. Monemar, J. Appl. Phys. 93, 126-133 (2003).

[21] S. J. Lim, S. Kwon, and H. Kim, Thin Solid Films 516, 1523-1528 (2008). 